\title{
Sudden stoppage of rotor in a thermally driven rotary motor made from double-walled carbon nanotubes
}

\author{
K. Cai ${ }^{1}$, J. Z. Yu ${ }^{1}$, H. Yin ${ }^{1}$, Q. H. Qin ${ }^{2, *}$ \\ ${ }^{1}$ College of Water Resources and Architectural Engineering, Northwest A\&F University, Yangling \\ 712100, China \\ ${ }^{2}$ Research School of Engineering, the Australian National University, Acton, ACT, 2601, Australia \\ *Corresponding author's email address: qinghua.qin@anu.edu.au (Q.H. Qin) \\ K. Cai's email address: caikun1978@163.com
}

\begin{abstract}
In a thermally driven rotary motor made from double-walled carbon nanotubes (DWCNT), the rotor (inner tube) can be actuated to rotate within the stator (outer tube) when the environmental temperature is high enough. A sudden stoppage of the rotor can occur when the inner tube has been actuated to rotate at a stable high speed. To find the mechanisms of such sudden stoppages, eight motor models with the same rotor but different stators are built and simulated in the canonical NVT ensembles. Numerical results demonstrate that the sudden stoppage of the rotor occurs when the difference between radii is near $0.34 \mathrm{~nm}$ at a high environmental temperature. A smaller difference between radii does not imply easier activation of the sudden rotor stoppage. During rotation, the positions and electron density distribution of atoms at the ends of the motor show that a $s p^{1}$ bonded atom on the rotor is attracted by the $s p^{1}$ atom with the biggest deviation of radial position on the stator, after which they become two $s p^{2}$ atoms. The strong bond interaction between the two atoms leads to the loss of rotational speed of the rotor within 1 ps. Hence, the sudden stoppage is attributed to two factors: the deviation of radial position of atoms at the stator's ends and the drastic thermal vibration of atoms on the rotor in rotation. For a stable motor, sudden stoppage could be avoided by reducing deviation of the radial position of atoms at the stator's ends. A nanobrake can be, thus, achieved by adjusting a $s p^{1}$ atom at the ends of stator to stop the rotation of rotor quickly.
\end{abstract}

Keywords: carbon nanotube, molecular dynamics, thermally driven rotation, double-walled carbon nanotubes

\section{Introduction}

Due to the ultralow friction between adjacent shells in multiwalled carbon nanotubes (MWCNTs), relative motion between the shells is easily actuated. Many nanodevices made from MWCNTs or DWCNTs, such as bearings and motors, have been developed in consideration of that unique property[1-5] and other special properties[6-8]. Vacek and Michl [9] suggested three possible 
methods to drive the rotation of a nanomotor, namely electric field[10, 11], gas or liquid flow[12], and light absorption[13, 14]. These methods have also been adopted in the design of CNT-based nanomotors. For example, Tu and $\mathrm{Hu}[15]$ designed a rotary motor driven by axially varying voltage under an isothermal condition. Wang et al.[16] built a rotary motor with one CNT shaft and three or six blades. The rotor in the motor rotated when it was actuated by an external electric field. Hamdi et al.[17] investigated a direct current driven rotary nanomotor made from MWCNTs. By controlling the density and flow rate of fluidic gases, Kang and Hwang[18] simulated the actuation of high-speed rotation of a CNT-based motor. Its rotational speed was around 1.5rad/ps, which means that the rotational frequency was about 240GHz. Prokop et al.[19] studied the behaviour of a gigahertz rotary molecular motor driven by either electric field or gas flow.

Besides the above methods for actuating rotation, environmental temperature can also drive the rotation of a CNT-based motor. In studying the large amplitude oscillation along the axis of the inner tube in a DWCNT driven by temperature, Xu and Zheng[20] found that the inner tube also had a rotational speed. At $100 \mathrm{~K}$, the angular velocity was about $0.04 \mathrm{rad} / \mathrm{ps}$, i.e., a rotational frequency of about $6.3 \mathrm{GHz}$. Cai et al.[21] investigated the mechanism of a thermally driven rotary motor based on DWCNT. They explained that the rotation of the inner tube in a fixed outer tube at a high environmental temperature is driven by the vibration of atoms on the inner tube and that the interaction between the two shells created a non-equilibrium torque before the rotational speed approaching a stable peak value. In a microcanonical NVE ensemble, if there is no degree of freedom (DOF) constraint applied on the motor, damping rotation of the rotor appears. As the system in a canonical NVT ensemble, both the momentum and the moment of momentum of system were not conservative. In the canonical NVT ensemble, to maintain an averagely constant temperature, the energy dissipation of the system due to the friction between two tubes is repaired by environmental thermal energy. It is also known that a higher relative motion between tubes leads to greater friction between the tubes. When the energy dissipation (due to friction) and absorption (from the environment) reached an equilibrium state, the DWCNT-based motor system worked in a stable state. Thus, the dynamic behaviour of the rotor was actually an intrinsic property of the system in its environment. Here we call the stable rotating state of system as the "coordinate state". In the report by Cai et al.[21], the rotational frequency of the inner tube in a fixed outer tube almost exceeded $160 \mathrm{GHz}$. In the present work, the frequency is even higher than $260 \mathrm{GHz}$.

After finding the feasibility of designing a rotary motor driven by uniform temperature[21], the way to design a stable motor becomes important. In this study, sudden stoppage of an inner tube in a fixed outer tube is found and the reasons are presented. The effects of asymmetry of the outer tube (stator of motor) on sudden stoppage are considered. For further comparisons, the difference in radii between the two tubes in the motor is also involved in simulation by a computational molecular dynamic (CMD) method. These results can be taken as guidance in design of a stable rotary motor or a nanobrake.

\section{Models and Method}

In the present CMD simulations, the adaptive intermolecular reactive empirical bond order (AIREBO) potential[22] is adopted to describe the force field of both covalent bonds and 
long-range van der Waals interaction among carbon atoms in a system. The whole system is under a canonical NVT ensemble. Before simulation, a Nosé-Hoover heat bath at a specified temperature is applied to the system. After 400 ps of relaxation, all the atoms on the outer tube are fixed while the atoms on the inner tube have no DOF constraints. In simulation, the time step size is $1 \mathrm{fs}$. In parallel computation, only 2 cores are adopted in the calculation to reduce errors caused by multiple cores. The classical molecular dynamics code of LAMMPS[23] is used in all the following simulations. The local electron density distribution is calculated using Quantum Espresso[24].

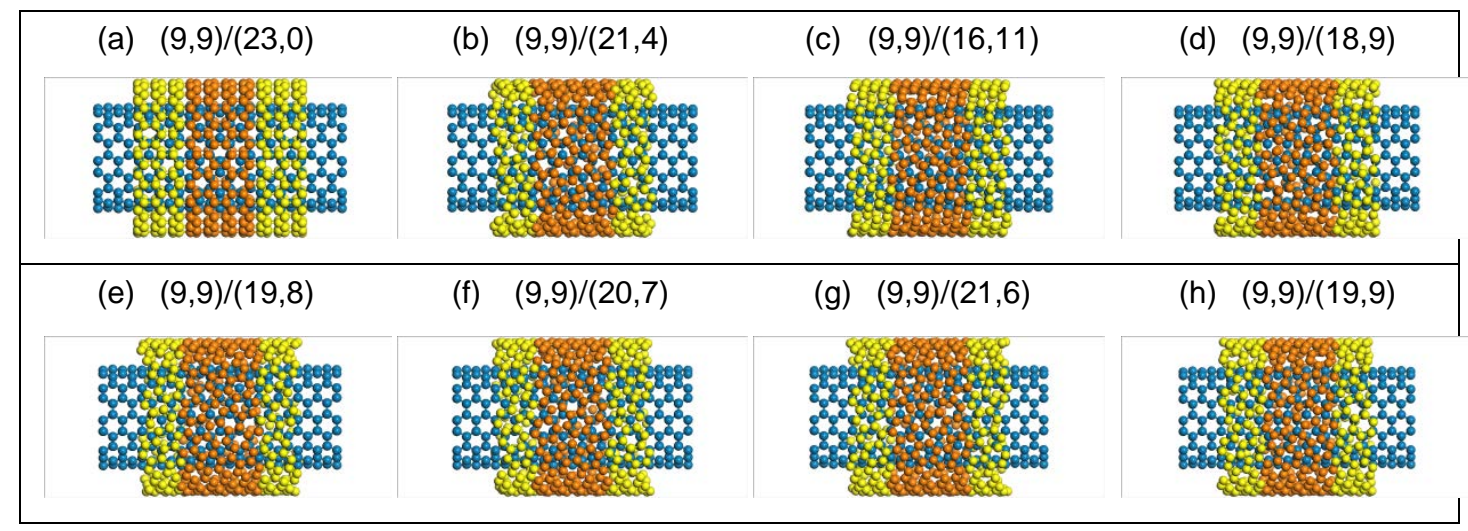

Fig. 1 Eight motor models from DWCNTs with the same rotor, i.e., $(9,9)$ but different stators.

Table 1 Parameters and of DWCNTs motor models in Fig. 1

\begin{tabular}{clccc}
\hline Model No. & \multicolumn{1}{c}{ Chirality } & Radii difference $(\mathrm{nm})$ & Lengths $(\mathrm{nm})$ & Number of atoms \\
\cline { 2 - 4 } (a) & $(9,9) /(23,0)$ & 0.295 & $2.993 / 2.016$ & $450 / 460$ \\
(b) & $(9,9) /(21,4)$ & 0.305 & $2.993 / 2.003$ & $450 / 422$ \\
(c) & $(9,9) /(16,11)$ & 0.315 & $2.993 / 1.972$ & $450 / 428$ \\
(d) & $(9,9) /(18,9)$ & 0.325 & $2.993 / 1.987$ & $450 / 450$ \\
(e) & $(9,9) /(19,8)$ & 0.335 & $2.993 / 2.011$ & $450 / 442$ \\
(f) & $(9,9) /(20,7)$ & 0.345 & $2.993 / 2.006$ & $450 / 444$ \\
(g) & $(9,9) /(21,6)$ & 0.355 & $2.993 / 2.050$ & $450 / 462$ \\
(h) & $(9,9) /(19,9)$ & 0.365 & $2.993 / 1.975$ & $450 / 454$ \\
\hline
\end{tabular}

Eight motor models made from DWCNT are built with different radii (see Fig. 1); the parameters are given in Table 1 . The yellow atoms at both ends of the stator are fixed alternatively for $200 \mathrm{ps}$ of relaxation. The deviation of the atoms on the outer tubes is enlarged by applying a non-coaxial inner tube in relaxation. Five environmental temperatures, 100, 300, 500, 800, and $1000 \mathrm{~K}$, are considered in the simulation.

\section{Results and Discussion}

Fig. 2 shows that the difference in radii between the two tubes has an obvious influence on the rotation of the inner tube. For example, when the difference is only $0.295 \mathrm{~nm}$ (Fig. 2a), rotation of the inner tube is barely actuated. The reason is that the small distance between the two walls leads to a higher potential barrier and the system cannot reach a coordinate state. Among all the models, the highest rotational speed of the inner tube occurs when the difference between radii is $0.325 \mathrm{~nm}$ 
(green curve in Fig. 2d), which is very close to the equilibrium distance between graphite sheets $(0.335 \mathrm{~nm})$. In Fig. 2e, the inner tube is excited to rotate at $100 \mathrm{~K}$, but that is the only instance among the total of 40 cases. That is to say, at low temperatures, rotation of the rotor is difficult to actuate. Fig. 2 further shows that in each model, the peak value of rotation of the inner tube depends on the environmental temperature. For example, the highest rotational speed of the inner tube in $(9,9) /(21,4)$ (Fig. 2b) is around $100 \mathrm{GHz}$ at different temperatures of $300 \mathrm{~K}$ (red curve) and $1000 \mathrm{~K}$ (green curve). In the model (9, 9)/(18, 9), the peak value is about $260 \mathbf{~ G H z}$ at $1000 \mathrm{~K}$ (green curve in Fig. 2d). It is also the highest among the total 40 cases. In the model $(9,9) /(21,6)$, the peak value is about $220 \mathrm{GHz}$ at $800 \mathrm{~K}$ (pink curve in Fig. 2g). The peak value is around 200 $\mathrm{GHz}$ at $500 \mathrm{~K}$ (blue curve in Fig. 2h). Thus, a higher temperature more easily excites the rotation of the inner tube. But the peak value of rotational speed of the rotor may not occur at the highest temperature for the same motor. Sometimes, the highest temperature barely actuates the rotation of the rotor (see the green curves in Fig. 2c, $f$ and g). The reason needs to be found.

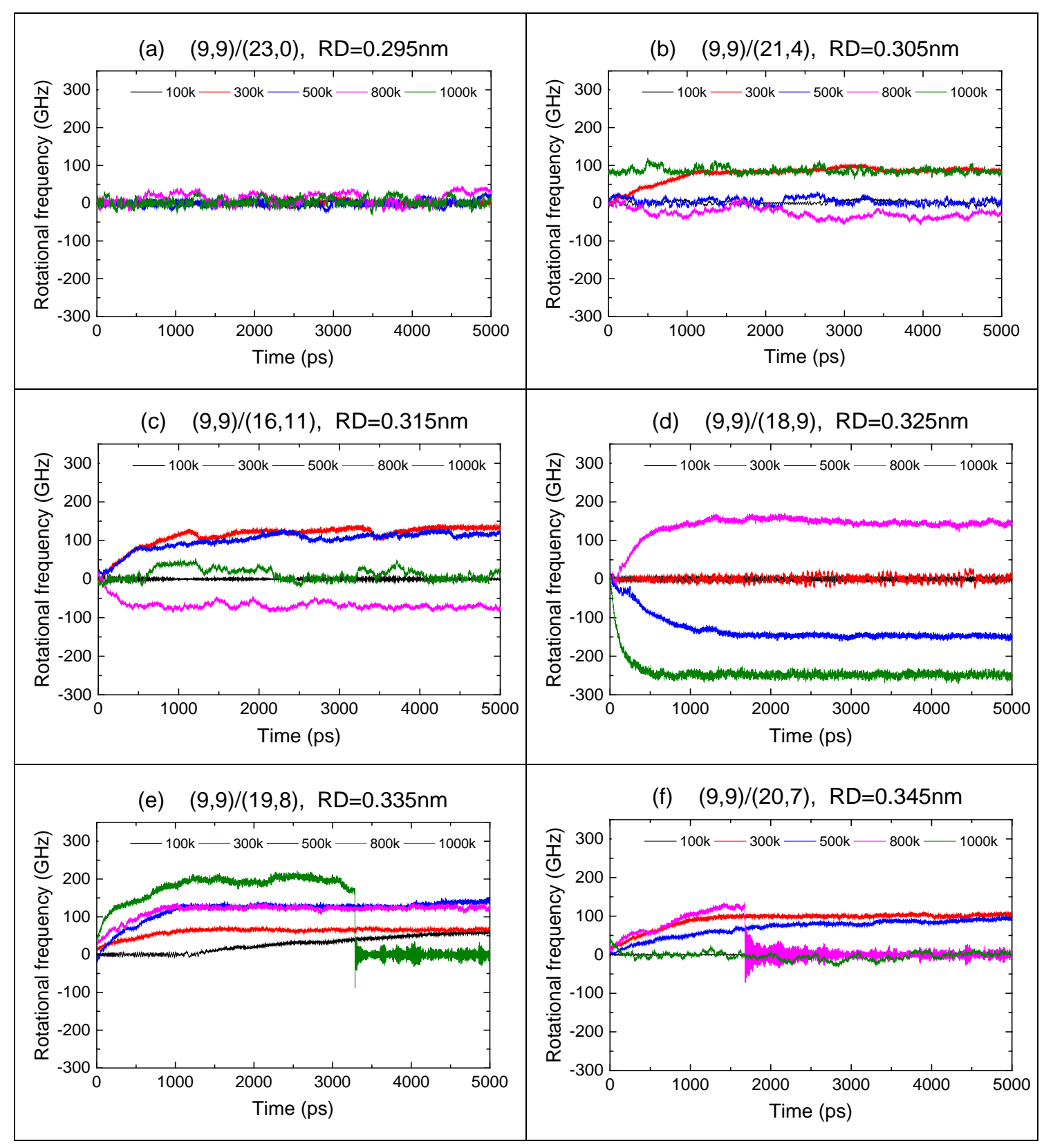




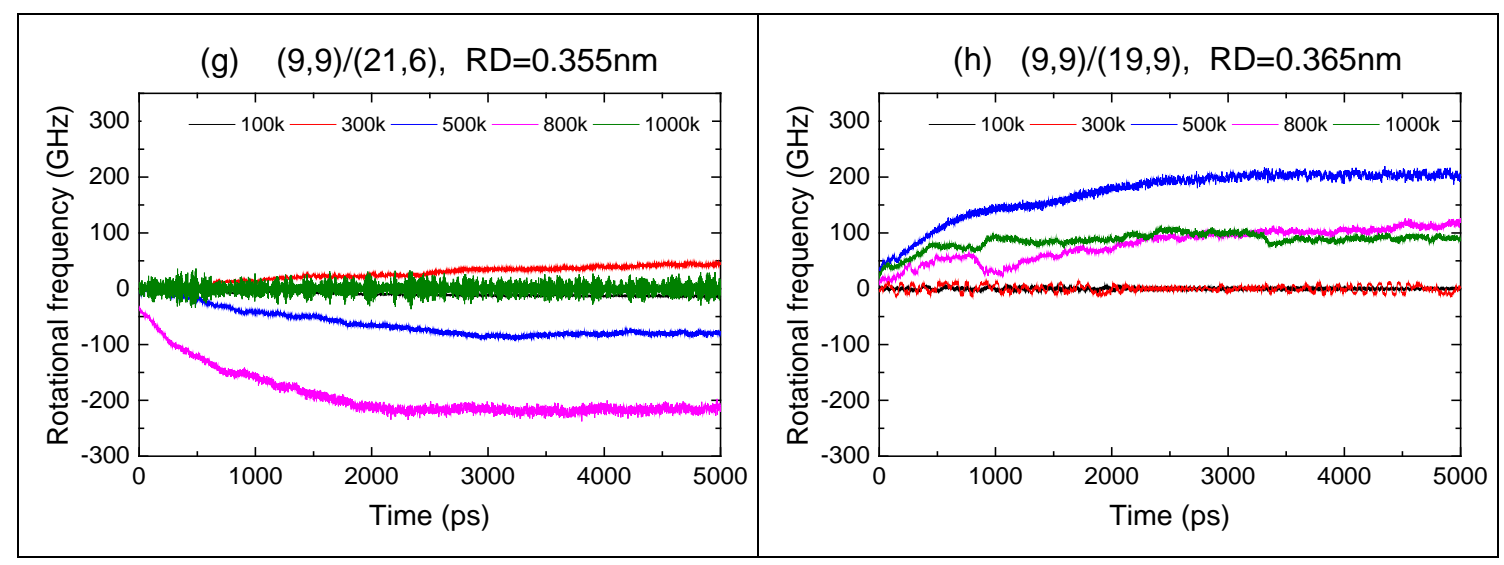

Fig. 2 Histories of rotational frequencies of inner tubes in motors with various differences between radii (RD) (100K-black curve, 300K-red curve, 500K-blue curve, 800K-pink curve, 1000K-green curve). Positive values indicate anticlockwise rotation of the inner tube along the axis in the left to right direction. Negative values indicate clockwise rotation.

In Figs. 2e and 2f, sudden stoppage of the rotor occurs during simulation as seen in the green curve $(1000 \mathrm{~K})$ at the $3285^{\text {th }}$ ps in Fig. 2e and in the pink curve $(800 \mathrm{~K})$ at the $1679^{\text {th }}$ ps in Fig. $2 \mathrm{f}$. Fig. 3 shows the positions of the two tubes in $(9,9) /(20,7)$ before and after the sudden stoppage of inner tube. It can be found from Fig. 3a that the yellow atom $\left(s p^{1}\right)$ on the stator deviates from the equilibrium position (the grey circle) more than the other atoms at the end of the stator. Hence the attraction between the yellow atom and the rotor must be higher than that of the other atoms on the stator. At the $1671^{\text {st }}$ ps, the two yellow atoms are close each other but the distance is not small enough as we toought. So, the rotor keeps stable rotation until $1678^{\text {th }}$ ps. Before the $1679^{\text {th }}$ ps, the two yellow atoms on the rotor (blue atoms) and the stator (pink atoms) are separate. From the configurations of the left end of motor at $1679^{\text {th }}$, the $1680^{\text {th }}$ and the $1681^{\text {st }} \mathrm{ps}$, we find that the inner tube shaves close to the yellow atom on the outer tube. It demonstrates that the rotor has no rotational speed on the time average.

Fig. 4 gives the electron density distribution on the cross-section of the left end of motor. At the $1677^{\text {th }}$ ps, the two tubes have no overlap of high electron density (Fig. 4a). At the $1678^{\text {th }}$ ps, the overlap of high electron density appears (Fig. 4b), whileas the overlap doesn't happen between the two marked atoms. As the rotational speed of rotor is high and the distance between the rotor and stator is not small enough, new covalent bond doesn't appear. So, the relative motion of the two tubes still exists. At the $1679^{\text {th }}$ ps, the overlap of higher electron density near the two marked atoms appears (Fig. 4c). The overlap continues in the subsequent simulation (Fig. 4d). As a result, we conclude that the two marked atoms ( $s p^{1}$ atoms) are bonded into two $s p^{2}$ atoms.

However, a lower difference between radii does not necessarily mean that the sudden stoppage ofthe rotor happens more easily. For example, there is no sudden stoppage in the models in Fig. 2(a-d), mainly because of the higher stable mutual repulsion between rotor and stator. Thermal vibration of atoms on the rotor is confined within a smaller region. The amplitude of the inward radial oscillation is greater than that of the outward oscillation. Therefore, a sudden stoppage is unlikely to occur.

If the system acts as a motor to drive the motion of other components in a NEMS (Nano electromechanical system), sudden stoppages should be avoided by reducing the maximum 
deviation from the equilibrium positions of both ends of the stator. On the other hand, to stop the rotation of the rotor quickly, we can adjust the deviation of an atom at the end of stator to form a covalent bond with an atom at the end of the rotor.

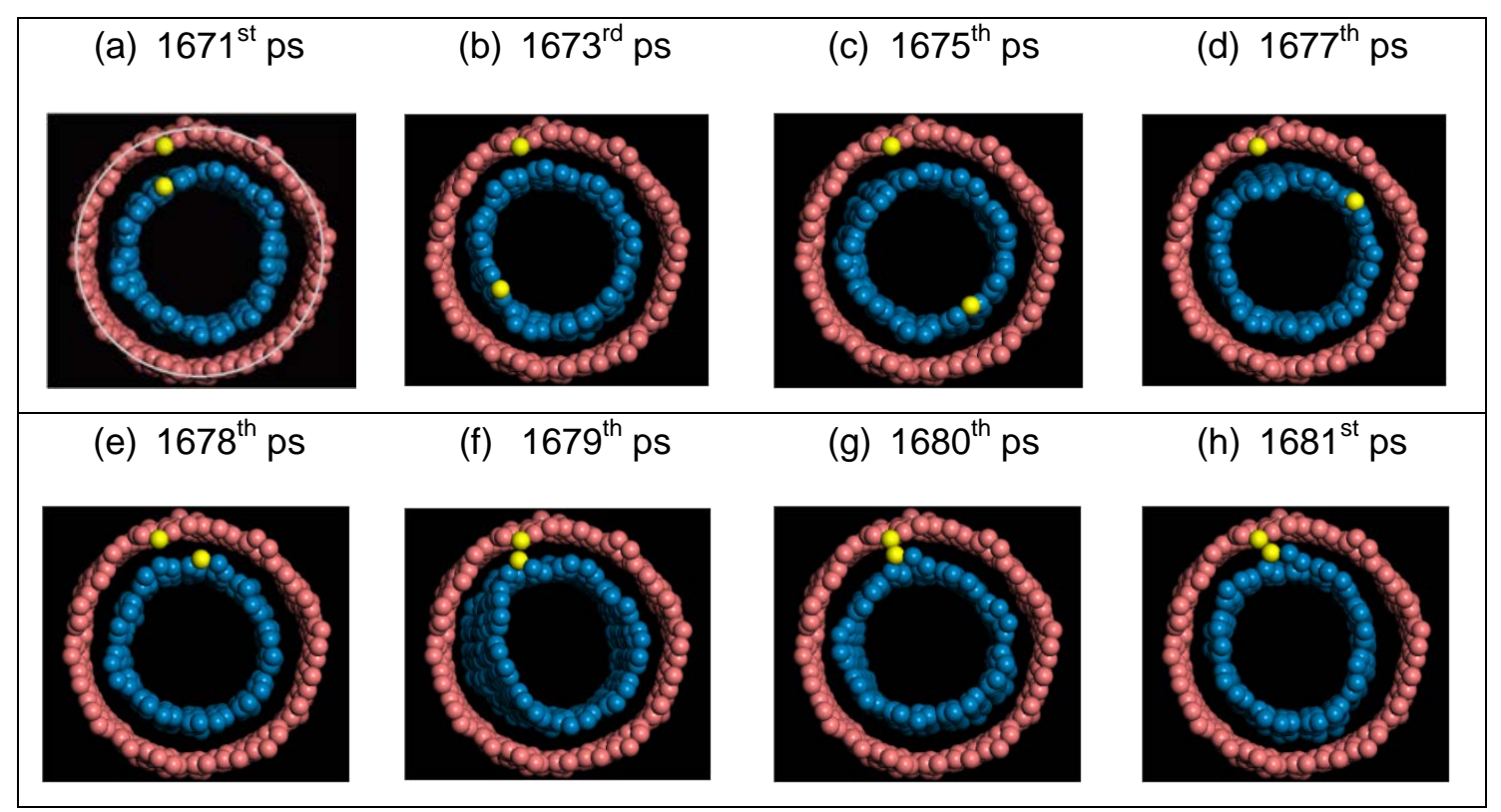

Fig. 3 Snapshots of the left end of system before and after sudden stoppage of rotor in $(20,7)$ stator. The grey circle in (a) represents the ideal symmetric boundary of the left end of the stator.

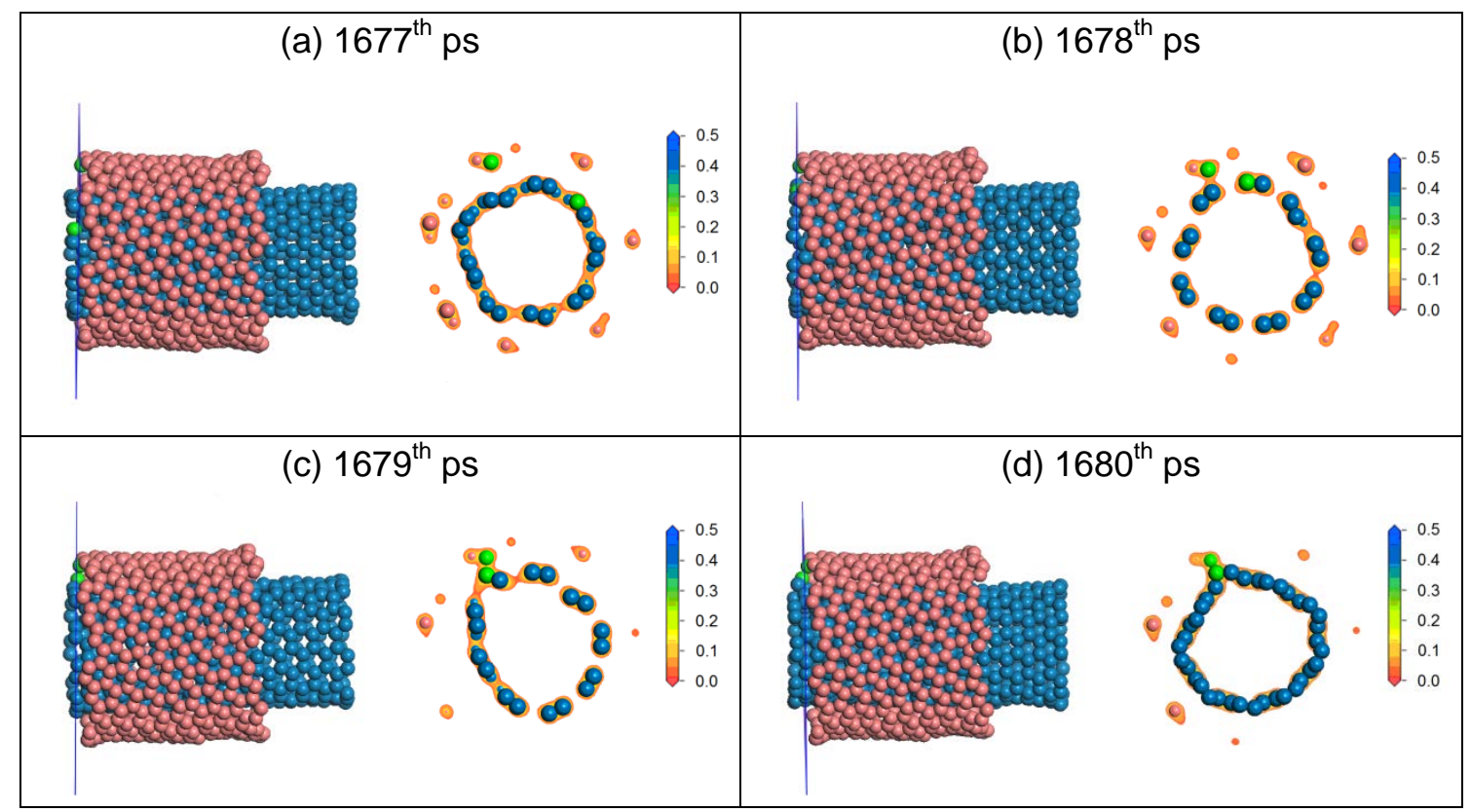

Fig. 4 Electron density distributions at the left end of $(9,9) /(20,7)$ motor before and after sudden stoppage.

\section{Conclusions}

From our numerical results, the sudden stoppage of rotor is identified and the dependence of sudden stoppage on the difference between radii and on environmental temperature is 
demonstrated. Some conclusions can be drawn for the potential application of nanomotors in NEMS.

1) In a DWCNT-based motor with a small difference in radii between two tubes or at low environmental temperature, excitation of the rotor is difficult, and no sudden stoppage occurs.

2) At high temperature, sudden stoppage of the rotor can easily occur in a motor with the difference of $\sim 0.34 \mathrm{~nm}$ between radii when the maximum radial deviation of atoms at the end of the outer tube is high.

3) In an oblique chiral outer tube, radial deviation of atom position on the outer tube is easily obtained. To maintain a stable rotating state of the thermally driven motor, such deviation should be controlled within a small range.

4) The phenomenon of sudden stoppage of the rotor also suggests another way to stop the rotation of an inner tube in a nanomotor, rather than relying on intertube friction.

\section{Acknowledgements}

The authors are grateful for financial support from the National Natural-Science-Foundation of China (Grant Nos. 50908190, 11372100).

\section{References}

[1] Forro, L., 2000 Nanotechnology - Beyond Gedanken experiments. Science, 289 560-1.

[2] Fennimore, A.M., T.D. Yuzvinsky, W.Q. Han, M.S. Fuhrer, J. Cumings, and A. Zettl, 2003 Rotational actuators based on carbon nanotubes. Nature, 424 408-10.

[3] Longhurst, M.J. and N. Quirke, 2007 Temperature-driven pumping of fluid through single-walled carbon nanotubes. Nano Letters, 7 3324-8.

[4] Cook, E.H., M.J. Buehler, and Z.S. Spakovszky, 2013 Mechanism of friction in rotating carbon nanotube bearings. Journal of the Mechanics and Physics of Solids, 61 652-73.

[5] Cai, K., H. Yin, Q.H. Qin, and Y. Li, 2014 Self-excited Oscillation of Rotating Double-walled Carbon Nanotubes. Nano letters, 14 2558-62.

[6] Qin, Z., Q.H. Qin, and X.-Q. Feng, 2008 Mechanical property of carbon nanotubes with intramolecular junctions: Molecular dynamics simulations. Physics Letters A, 372 6661-6.

[7] Qiu, W., Y. Kang, Z. Lei, Q.H. Qin, and Q. Li, 2009 A new theoretical model of a carbon nanotube strain sensor. Chinese Physics Letters, 26080701.

[8] Qiu, W., Y.L. Kang, Z.K. Lei, Q.H. Qin, Q. Li, and Q. Wang, 2010 Experimental study of the Raman strain rosette based on the carbon nanotube strain sensor. Journal of Raman Spectroscopy, 41 1216-20.

[9] Vacek, J. and J. Michl, 2007 Artificial surface-mounted molecular rotors: Molecular dynamics simulations. Advanced Functional Materials, 17 730-9.

[10] Zheng, X.L., M.E. Mulcahy, D. Horinek, F. Galeotti, T.F. Magnera, and J. Michl, 2004 Dipolar and nonpolar altitudinal molecular rotors mounted on an $\mathrm{Au}(111)$ surface. Journal of the American Chemical Society, 126 4540-2.

[11] Horinek, D. and J. Michl, 2003 Molecular dynamics simulation of an electric field driven dipolar molecular rotor attached to a quartz glass surface. Journal of the American Chemical Society, 125 11900-10. 
[12] Vacek, J. and J. Michl, 1997 A molecular "Tinkertoy" construction kit: Computer simulation of molecular propellers. New Journal of Chemistry, 21 1259-68.

[13] Feringa, B.L., 2001 In control of motion: From molecular switches to molecular motors. Accounts of Chemical Research, 34 504-13.

[14] Leigh, D.A., J.K.Y. Wong, F. Dehez, and F. Zerbetto, 2003 Unidirectional rotation in a mechanically interlocked molecular rotor. Nature, 424 174-9.

[15] Tu, Z.C. and X. Hu, 2005 Molecular motor constructed from a double-walled carbon nanotube driven by axially varying voltage. Physical Review $B, 72$.

[16] Wang, B., L. Vukovic, and P. Kral, 2008 Nanoscale Rotary Motors Driven by Electron Tunneling. Physical Review Letters, 101.

[17] Hamdi, M., A. Subramanian, L. Dong, A. Ferreira, and B.J. Nelson, 2013 Simulation of Rotary Motion Generated by Head-to-Head Carbon Nanotube Shuttles. Ieee-Asme Transactions on Mechatronics, 18 130-7.

[18] Kang, J.W. and H.J. Hwang, 2004 Nanoscale carbon nanotube motor schematics and simulations for micro-electro-mechanical machines. Nanotechnology, 15 1633-8.

[19] Prokop, A., J. Vacek, and J. Michl, 2012 Friction in Carborane-Based Molecular Rotors Driven by Gas Flow or Electric Field: Classical Molecular Dynamics. Acs Nano, 6 1901-14.

[20] Xu, Z., Q.-S. Zheng, and G. Chen, 2007 Thermally driven large-amplitude fluctuations in carbon-nanotube-based devices: Molecular dynamics simulations. Physical Review B, 75 195445.

[21] Cai, K., Y. Li, Q.H. Qin, and Y. Yin, 2014 Gradientless temperature-driven rotating motor from a double-walled carbon nanotube. Nanotechnology, 25505701.

[22] Stuart, S.J., A.B. Tutein, and J.A. Harrison, 2000 A reactive potential for hydrocarbons with intermolecular interactions. Journal of Chemical Physics, 112 6472-86.

[23] LAMMPS Molecular Dynamics Simulator. (http://lammps.sandia.gov/, 2013).

[24] Materials Studio. (http://accelrys.com/products/materials-studio/, 2013). 